Pacific Journal of Mathematics

THE GROUP OF AUTOMORPHISMS OF THE HOLOMORPH
OF A GROUP

OF 


\title{
THE GROUP OF AUTOMORPHISMS OF THE HOLOMORPH OF A GROUP
}

\author{
NAI-CHAO HSU
}

1. Introduction. If $G=H K$ where $H$ is a normal subgroup of the group $G$ and where $K$ is a subgroup of $G$ with the trivial intersection with $H$, then $G$ is said to be a semi-direct product of $H$ by $K$ or a splitting extension of $H$ by $K$. We can consider a splitting extension $G$ as an ordered triple $(H, K ; \phi)$ where $\phi$ is a homomorphism of $K$ into the automorphism group $\mathfrak{A}(H)$ of $H$. The ordered triple $(H, K ; \phi)$ is the totality of all ordered pairs $(h, k), h \in H, k \in K$, with the multiplication

$$
(h, k)\left(h^{\prime}, k^{\prime}\right)=\left(h \phi_{k}\left(h^{\prime}\right), k k^{\prime}\right) .
$$

If $\phi$ is a monomorphism of $K$ into $\mathfrak{A}(H)$, then $(H, K ; \phi)$ is isomorphic to $(H, \phi(K) ; \iota)$ where $\iota$ is the identity mapping of $\phi(K)$, and therefore $G$ is the relative holomorph of $H$ with respect to a subgroup $\phi(K)$ of $\mathfrak{U}(H)$. If $\phi$ is an isomorphism of $K$ onto $\mathfrak{A}(H)$, then $G$ is the holomorph of $H$.

Let $H$ be a group, and let $G$ be the holomorph of $H$. We are considering $H$ as a subgroup of $G$ in the usual way. Gol'fand [1] studied the group $\mathfrak{A}_{H}(G)$ of automorphisms of $G$ each of which maps $H$ onto itself, the group $\Im(G)$ of inner automorphisms of $G$, and the factor group $\mathfrak{A}_{H}(G) / \mathfrak{\Im}(G)$. In case $H$ is abelian, this factor group is isomorphic to the first cohomology group of $\mathfrak{A}(H)$ acting on $H$, as Mills [4] mentioned. In $\S 2$, we generalize Gol'fand's results by dealing with a relative holomorph instead of with the holomorph. The fact that $\phi$ is a monomorphism is essential for the proof. Hence this generalization of Gol'fand's theory is in some sense the best possible one. Gol'fand [1], Miller [3], Mills [4], Peremans [5] and Specht [6, pp. 101-102] discussed the group of automorphisms of the holomorph of some groups. In $\S 3$ and 4 , we discuss the group of automorphisms of the holomorph of some other uncomplicated groups. As applications we can describe the group of automorphisms of the holomorph of symmetric groups and the group of automorphisms of the holomorph of subgroups of the additive group of rational numbers.

We set up our basic device which determines all automorphisms of a splitting extension $G=(H, K ; \phi)$ in terms of mappings of $H$ and $K$. It also enables us to compute the product of two automorphisms of $G$.

Received July 25, 1960. This work was supported in part by the U. S. Air Force under a contract. It forms part of the author's thesis, Washington University, 1960, written under the direction of Professor F. Haimo, to whom the author wishes to express his gratitude for advice and encouragement. The author also wishes to express his thanks to the referee for comments. 
Lemma 1.1. Let $G=(H, K ; \phi)$ and let $\mathfrak{M}$ be the set of all quadruples $\left[\begin{array}{ll}A & C \\ B & D\end{array}\right]$, where $^{1} A \in \operatorname{Map}(H, H), B \in \operatorname{Map}(H, K), C \in \operatorname{Map}(K, H)$ and $D \in \operatorname{Map}(K, K)$ satisfying the following conditions:

(1.1.1) For all $h, h^{\prime} \in H, A\left(h h^{\prime}\right)=A(h) \phi_{B(h)}\left(A\left(h^{\prime}\right)\right)$.

(1.1.2) For all $h, h^{\prime} \in H, B\left(h h^{\prime}\right)=B(h) B\left(h^{\prime}\right)$.

(1.1.3) For all $k, k^{\prime} \in K, C\left(k k^{\prime}\right)=C(k) \phi_{D(k)}\left(C\left(k^{\prime}\right)\right)$.

(1.1.4) For all $k, k^{\prime} \in K, D\left(k k^{\prime}\right)=D(k) D\left(k^{\prime}\right)$.

(1.1.5) For all $h \in H, k \in K, B\left(\phi_{k}(h)\right) D(k)=D(k) B(h)$.

(1.1.6) For all $h \in H, k \in K, A\left(\phi_{k}(h)\right) \phi_{B\left(\phi_{k}(h)\right)}(C(k))=C(k) \phi_{D(k)}(A(h))$.

(1.1.7) For any $\left(h^{\prime}, k^{\prime}\right) \in G$, there exists a unique $(h, k) \in G$ satisfying $\left(A(h) \phi_{B(h)}(C(k)), B(h) D(k)\right)=\left(h^{\prime}, k^{\prime}\right)$.

Then there is a one-to-one correspondence between the automorphism group $\mathfrak{A}(G)$ of $G$ and $\mathfrak{M}$ under the correspondence $\alpha \rightarrow\left[\begin{array}{ll}A & C \\ B & D\end{array}\right]$ defined by

$$
\alpha(h, e)=(A(h), B(h)) \quad \text { and } \quad \alpha(e, k)=(C(k), D(k)) .
$$

Further, if

$$
\alpha \rightarrow\left[\begin{array}{ll}
A_{\alpha} & C_{\alpha} \\
B_{\alpha} & D_{\alpha}
\end{array}\right], \quad \beta \rightarrow\left[\begin{array}{cc}
A_{\beta} & C_{\beta} \\
B_{\beta} & D_{\beta}
\end{array}\right] \quad \text { and } \quad \beta \alpha \rightarrow\left[\begin{array}{cc}
A_{\beta \alpha} & C_{\beta \alpha} \\
B_{\beta \alpha} & D_{\beta \alpha}
\end{array}\right],
$$

where $\beta \alpha$ denotes the automorphism, arising by first applying $\alpha$ and then $\beta$, then

$$
\begin{aligned}
& A_{\beta \alpha}(h)=A_{\beta}\left(A_{\alpha}(h)\right) \phi_{B_{\beta}\left(A_{\alpha}(h)\right)}\left(C_{\beta}\left(B_{\alpha}(h)\right)\right), \\
& B_{\beta \alpha}(h)=B_{\beta}\left(A_{\alpha}(h)\right) D_{\beta}\left(B_{\alpha}(h)\right) \\
& C_{\beta \alpha}(k)=A_{\beta}\left(C_{\alpha}(k)\right) \phi_{B_{\beta}\left(\sigma_{\alpha}(k)\right)}\left(C_{\beta}\left(D_{\alpha}(k)\right)\right)
\end{aligned}
$$

and

$$
D_{\beta \alpha}(k)=B_{\beta}\left(C_{\alpha}(k)\right) D_{\beta}\left(D_{\alpha}(k)\right) .
$$

Because of the one-to-one correspondence described above, we identify an element of $\mathfrak{A}(G)$ with the corresponding element of $\mathfrak{M}$. As the multiplication formula in $\mathfrak{A}(G)$ we have

$$
\left[\begin{array}{ll}
A_{\beta} & C_{\beta} \\
B_{\beta} & D_{\beta}
\end{array}\right]\left[\begin{array}{ll}
A_{\alpha} & C_{\alpha} \\
B_{\alpha} & D_{\alpha}
\end{array}\right]=\left[\begin{array}{ll}
A_{\beta} A_{\alpha}+\phi_{B_{\beta} A_{\alpha}} C_{\beta} B_{\alpha} & A_{\beta} C_{\alpha}+\phi_{B_{\beta} \sigma_{\alpha}} C_{\beta} D_{\alpha} \\
B_{\beta} A_{\alpha}+D_{\beta} B_{\alpha} & B_{\beta} C_{\alpha}+D_{\beta} D_{\alpha}
\end{array}\right] .
$$

Let $X$ be a group and $Y$ a subgroup of $X$. By $\langle x\rangle_{x}$, we denote the inner automorphism of $X$ induced by $x \in X$. By $\langle x\rangle_{X} / Y$, we denote the restriction of $\langle x\rangle_{X}$ to $Y$. By $Z(X)$ and $N(Y, X)$, we denote the center of $X$ and the normalizer of $Y$ in $X$, respectively.

1 If $X$ and $Y$ are two groups, by $\operatorname{Map}(X, Y)$ is meant the set of all mappings of $X$ into $Y$. The same sort of self-explanatory notations such as $\operatorname{Hom}(X, Y)$, End $(X)$ will also be employed. 

$\mathfrak{U}(H)$.

In the following definitions ${ }^{2}$, let $H$ be a group and $K$ a subgroup of

A mapping $U$ of $K$ into $H$ is called a crossed character of $K$ into $H$ if and only if, for all $k, k^{\prime} \in K$,

$$
U\left(k k^{\prime}\right)=U(k)\left(U\left(k^{\prime}\right)\right)^{k} \text {. }
$$

Here and hereafter a symbol like $h^{k}$ is used as substitute for $k(h)$.

A crossed character $U$ of $K$ into $H$ is called regular if and only if the correspondence

$$
k \rightarrow\langle U(k)\rangle_{H} k
$$

is an automorphism of $K$. That $\langle U(k)\rangle_{H} \in K$ for every $k \in K$ is an implicit requirement of the definition.

A mapping $U$ of $K$ into $H$ is called a principal character of $K$ into $H$ if and only if there exists $h \in H$ such that for all $k \in K$

$$
U(k)=h\left(h^{-1}\right)^{k} .
$$

In this case, $U$ is said to be defined by $h$.

A principal character $U$ of $K$ into $H$ defined by $h \in H$ is called regular if and only if

$$
\langle h\rangle_{H} \in N(K, \mathfrak{A}(H)) \text {. }
$$

A principal character $U$ of $K$ into $H$ defined by $h \in H$ is called superregular if and only if

$$
\langle h\rangle_{H} \in K \text {. }
$$

By $\sqrt{5}$ and $\mathbb{C}^{r}$, we denote the set of all crossed characters of $K$ into $H$ and the set of all regular crossed characters of $K$ into $H$, respectively. $\mathfrak{P}, \mathfrak{P}^{r}$ and $\mathfrak{P}^{s}$, we denote the set of all principal characters of $K$ into $H$, the set of all regular principal characters of $K$ into $H$ and the set of all superregular principal characters of $K$ into $H$, respectively.

The following facts are readily verified.

Proposition 1.2. Every principal character of $K$ into $H$ is a crossed character of $K$ into $H$.

Proposition 1.3. Let $U$ be a principal character of $K$ into $H$. Then $U$ is a regular principal character of $K$ into $H$ if and only if $U$ is a regular crossed character of $K$ into $H$.

2. Gol'fand's theory for a relative holomorph. By $E$ and 0 , we denote the identity mapping and the trivial mapping, respectively, with 
suitable domain and range. By specializing $\phi=\iota, B=0$ and $C=0$ in (1.1), we have

Proposition 2.1. Let $G=(H, K ; \iota)$. Then $\left[\begin{array}{cc}A & 0 \\ 0 & D\end{array}\right] \in \mathfrak{A}(G)$ if and only if

$$
A \in N(K, \mathfrak{U}(H)) \quad \text { and } \quad D=\langle A\rangle_{N} / K
$$

hold, where $N$ stands for $N(K, \mathfrak{A}(H))$.

By $\mathfrak{A}_{H, K}(G)$, we denote the set of all automorphisms of $G$ each of which maps each of $H$ and $K$ onto themselves. It follows from (2.1) that

Proposition 2.2. Let $G=(H, K ; \iota)$. Then

$$
\mathfrak{U}_{H, K}(G) \cong N(K, \mathfrak{A}(H))
$$

under the mapping $\left[\begin{array}{cc}A & 0 \\ 0 & D\end{array}\right] \rightarrow A$.

On the other hand, by specializing $\phi=\iota, A=E$ and $B=0$ in (1.1), we have

Proposition 2.3. Let $G=(H, K ; \iota)$. Then $\left[\begin{array}{cc}E & C \\ 0 & D\end{array}\right] \in \mathfrak{A}(G)$ if and only if the following conditions are satisfied:

(2.3.1) $D \in \mathfrak{A}(K)$.

(2.3.2) $C D^{-1} \in \mathfrak{( 5}^{r}$.

(2.3.3) $\left\langle C D^{-1}(k)\right\rangle_{H} k=D^{-1}(k)$ for all $k \in K$.

Proposition 2.4. Let $G=(H, K ; \iota)$. Then, for any $U \in \mathbb{C}^{r}$, there exists a unique $\left[\begin{array}{cc}E & C \\ 0 & D\end{array}\right] \in \mathfrak{A}(G)$ such that $C D^{-1}=U$.

Proof. For any given $U \in \mathfrak{C}^{r}$, we define an automorphism $D^{-1}$ of $K$ by

$$
D^{-1}(k)=\langle U(k)\rangle_{H} \mathrm{k}
$$

and define a mapping $C$ of $K$ into $H$ by $C=U D$. Then by (2.3) we see that $\left[\begin{array}{cc}E & C \\ 0 & D\end{array}\right] \in \mathfrak{A}(G)$, proving the existence. By (2.3.3) and by our definition we have the uniqueness.

By ${ }_{H} \mathfrak{A}(G)$, we denote the set of all automorphisms of $G$ each of which is an extension of the identity mapping on $H$.

Proposition 2.5. Let $G=\left(H, K ;\right.$ ı). Then ${ }_{H} \mathfrak{H}(G)$ is isomorphic to (5) with the multiplication 


$$
\left(U_{1} \circ U\right)(k)=U\left(\left\langle U_{1}(k)\right\rangle_{H}\right) U_{1}(k) U(k)
$$

under the correspondence

$$
\left[\begin{array}{cc}
E & C \\
0 & D
\end{array}\right] \rightarrow C D^{-1}
$$

Proof. By (2.3) and (2.4), we see that the correspondence is a oneto-one mapping of ${ }_{H} \mathfrak{2}(G)$ onto $\mathbb{C}^{r}$. By the help of (2.3.3), we can show that it is an isomorphism.

Proposition 2.6. Let $G=(H, K ; \iota) . \quad$ If $\left[\begin{array}{cc}A & C \\ 0 & D\end{array}\right] \in \mathfrak{A}_{H}(G)$ and if $A \in N=N(K, \mathfrak{A}(H))$, then

$$
\begin{aligned}
& D \in \mathfrak{A}(K), \\
& {\left[\begin{array}{cc}
E & C\left\langle A^{-1}\right\rangle_{N} / K \\
0 & D\left\langle A^{-1}\right\rangle_{N} / K
\end{array}\right] \in \mathfrak{A}(G),} \\
& {\left[\begin{array}{cc}
A & 0 \\
0 & \langle A\rangle_{N} / K
\end{array}\right] \in \mathfrak{A}(G)}
\end{aligned}
$$

and

$$
\left[\begin{array}{cc}
A & C \\
0 & D
\end{array}\right]=\left[\begin{array}{cc}
E & C\left\langle A^{-1}\right\rangle_{N} / K \\
0 & D\left\langle A^{-1}\right\rangle_{N} / K
\end{array}\right]\left[\begin{array}{cc}
A & 0 \\
0 & \langle A\rangle_{N} / K
\end{array}\right]
$$

Proof. Specializing $\phi=\iota$ and $B=0$ in (1.1), we have $D \in \mathfrak{U}(K)$. By (2.1)

$$
\left[\begin{array}{cc}
A & 0 \\
0 & \langle A\rangle_{N} / K
\end{array}\right] \in \mathfrak{A}(G)
$$

and the rest follows immediately.

THEOREM 2.7. Let $G=(H, K$; ८). Suppose that, for every

$$
\left[\begin{array}{cc}
A & C \\
0 & D
\end{array}\right] \in \mathfrak{A}_{H}(G)
$$

we have $A \in N=N(K, \mathfrak{A}(H))$. Then

$$
\mathfrak{A}_{H}(G) \cong\left(\mathfrak{C}^{r}, N ; \phi\right)
$$

with $\phi \in \operatorname{Hom}\left(N, \mathfrak{A}\left(\mathbb{C}^{r}\right)\right)$ defined by

$$
\phi_{A}(U)=A U\left\langle A^{-1}\right\rangle_{N} / K
$$

for $A \in N$ and for $U \in \mathfrak{\complement}^{r}$. 
Proof. By means of (2.6), we can show that $\mathfrak{A}_{H}(G)$ is a splitting extension of ${ }_{H} \mathfrak{A}(G)$ by $\mathfrak{U}_{H, K}(G)$. By (2.5) and (2.2), we conclude the proof.

By (2.3.1) and (2.3.3), we have

Proposition 2.8. Let $G=(H, K ; \iota)$ and suppose $\left[\begin{array}{cc}E & C \\ 0 & D\end{array}\right] \in \mathfrak{U}(G)$. Then $\left[\begin{array}{cc}E & C \\ 0 & D\end{array}\right] \in \Im(G)$ if and only if $C D^{-1} \in \mathfrak{P}^{s}$.

By (1.3), (2.4) and (2.8), we have

Proposition 2.9. Let $G=(H, K, \iota)$. Then, for any $U \in \mathfrak{P}^{s}$, there exists a unique $\left[\begin{array}{cc}E & C \\ 0 & D\end{array}\right] \in \Im(G)$ such that $C D^{-1}=U$.

TheOREM 2.10. Let $G=(H, K ; \iota)$. Then $\mathfrak{P}^{s}$ is a normal subgroup of $\mathfrak{C}^{r}$

$$
{ }_{H} \mathfrak{A}(G) \cap \mathfrak{\Im}(G) \cong \mathfrak{P}^{s}
$$

and

$$
\frac{\mathfrak{\Im}^{r}}{\mathfrak{S}^{s}} \cong \frac{{ }^{\mathfrak{A}}(G) \mathfrak{I}(G)}{\mathfrak{J}(G)}
$$

Proof. In view of (2.8) and (2.9), the isomorphism (2.5.1) induces an isomorphism of ${ }_{\boldsymbol{H}} \mathfrak{A}(G) \cap \mathfrak{\Im}(G)$ onto $\mathfrak{H}^{s}$. The rest follows from this easily.

Let $G=(H, K ; \iota)$. By $\hat{K}$, we denote the set of all automorphisms of $H$ each of which can be extended to an inner automorphism of $G$ which maps $K$ onto itself. By definition and by virtue of (2.1), we can easily verify the following fact:

Proposition 2.11. Let $G=(H, K ; \iota)$. Then $\hat{K}$ is a subgroup of $\mathfrak{A}(H)$ lying between $K$ and $N(K, \mathfrak{A}(H))$. Further, every $A \in \hat{K}$ determines $\left[\begin{array}{cc}A & 0 \\ 0 & D\end{array}\right] \in \Im(G)$ uniquely, i.e., $D=\langle A\rangle_{N} / K$.

TheOREm 2.12. Let $G=(H, K ; \iota)$. Suppose that, for every

$$
\left[\begin{array}{cc}
A & C \\
0 & D
\end{array}\right] \in \Im(G)
$$

we have $A \in \hat{K}$. Then

$$
\Im(G) \cong\left(\mathfrak{P}^{s}, \hat{K} ; \phi\right)
$$

where $\phi \in \operatorname{Hom}\left(\hat{K}, \mathfrak{A}\left(\mathfrak{P}^{s}\right)\right)$ defined by $\phi_{A}(U)=A U\left\langle A^{-1}\right\rangle_{N} / K$. 
Proof. Let $\left[\begin{array}{cc}A & C \\ 0 & D\end{array}\right] \in \Im(G)$. Then, by (2.11), $A \in N(K, \mathfrak{A}(H))$, and therefore the decomposition (2.6.1) is available, from which it follows that

$$
\left[\begin{array}{ll}
E & C\left\langle A^{-1}\right\rangle_{N} / K \\
0 & D\left\langle A^{-1}\right\rangle_{N} / K
\end{array}\right]
$$

is an inner automorphism of $G$. Hence

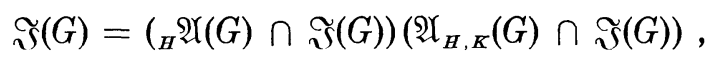

and it is easy to see that $\mathfrak{\Im}(G)$ is a splitting extension of ${ }_{\theta} \mathfrak{U}(G) \cap \Im(G)$ by $\mathfrak{U}_{H, K}(G) \cap \mathfrak{\Im}(G)$. By (2.10) and (2.11), we conclude the proof.

TheOREM 2.13. Let $G=(H, K ; \iota)$. Suppose that, for every

$$
\left[\begin{array}{ll}
A & C \\
0 & D
\end{array}\right] \in \mathfrak{U}_{H}(G),
$$

we have $A \in \hat{K}$. Then

$$
\frac{\mathfrak{S}^{r}}{\mathfrak{F}^{s}} \cong \frac{\mathfrak{M}_{H}(G)}{\mathfrak{S}(G)}
$$

Proof. Let $\left[\begin{array}{cc}A & C \\ 0 & D\end{array}\right] \in \mathfrak{U}_{H}(G)$. Then, by (2.11), $\left[\begin{array}{cc}A & 0 \\ 0 & \langle A\rangle_{N} / K\end{array}\right] \in \Im(G)$. The decomposition (2.6.1) is available, from which

$$
\mathfrak{U}_{H}(G)={ }_{H} \mathfrak{U}(G) \mathfrak{\Im}(G)
$$

follows. We conclude the proof by (2.10).

In case $H$ is abelian, the factor group mentioned in the preceding theorem is isomorphic to the first cohomology group of $K$ acting on $H$.

3. Application to the holomorph of a complete group.

Proposition 3.1. Let $G=(H, K$; ८) where $H$ is centerless, $K \subset \Im(H)$ and $\mathfrak{U}(K)=\mathfrak{S}(K)$. Then

$$
\mathfrak{C}^{r}=\mathfrak{P}^{s}
$$

Proof. By definition and by (1.3), $\mathfrak{P}^{s} \subset \mathfrak{C}^{r}$. Conversely, let $U \in \mathfrak{\complement}^{r}$. Then, by (2.4), there exists $\left[\begin{array}{cc}E & C \\ 0 & D\end{array}\right] \in \mathfrak{U}(G)$ such that $C D^{-1}=U$, where $D \in \mathfrak{A}(K)$ by (2.3). By assumption,

$$
D=\left\langle\left\langle d^{-1}\right\rangle_{H}\right\rangle_{\boldsymbol{K}}
$$

for some $d \in H$ with $\left\langle d^{-1}\right\rangle_{\boldsymbol{B}} \in K$. By (2.3.3) and by the fact that $H$ is centerless, we have 


$$
C D^{-}(k)=d\left(d^{-1}\right)^{k},
$$

which shows that $U \in \mathfrak{P}^{s}$, and therefore $\mathbb{C}^{r} \subset \mathfrak{P}^{s}$.

From this we have

Proposition 3.2. Let $G$ be the holomorph of a complete group $H$. Then

$$
\mathbb{C}^{r}=\mathfrak{P}^{8}
$$

This, together with (2.13), implies

THEOREM 3.3. Let $G$ be the holomorph of a complete group $H$. Then

$$
\mathfrak{A}_{H}(G)=\mathfrak{J}(G) \text {. }
$$

Since $G$ has no center, we have

COROLlaRY 3.4. Let $G$ be the holomorph of a complete group $H$. Then

$$
\mathfrak{A}_{H}(G) \cong G \text {. }
$$

THEOREM 3.5. ${ }^{3}$ Let $G$ be the holomorph of a finite complete group $H$ such that the square of the index of any non-trivial normal subgroup of $H$ is smaller than the order of $H$. Then $\mathfrak{A}(G)$ is a splitting extension of $\Im(G)$ by a group of order 2.

Proof. Since $H$ is a complete group and is a normal subgroup of $G$, we have $G=H \times K$ where $K$ is the centralizer of $H$ in $G$ [2, vol. 2, p. 80]. $K$ consists of all elements of the form $\left(h^{-1},\langle h\rangle_{H}\right)$ with $h \in H$. Obviously $K$ is isomorphic to $H$ under the mapping $\left(h^{-1},\langle h\rangle_{H}\right) \rightarrow(h, e)$. We shall represent every automorphism of $G$ with reference to $H \times K$ by means of (1.1) with $\phi$ trivial. Suppose that we are given

$$
\alpha=\left[\begin{array}{ll}
A & C \\
B & D
\end{array}\right] \in \mathfrak{A}(G)
$$

with $A \notin \Re(H)$. Since $H$ cannot be isomorphic to any of its proper subgroups, we have $\operatorname{ker} A \neq(e)$. If $B$ were not an isomorphism of $H$ onto $K$, then we would have either
(a) $B(H) \neq K$
and $\operatorname{ker} B=(e)$ or
(b) $\operatorname{ker} B \neq(e)$.

Since $H$ and $K$ are isomorphic and since $H$ cannot be isomorphic to any of its subgroups, (a) is impossible. On the other hand, (b) would imply

${ }^{3}$ The referee pointed out that this theorem is an easy consequence of the Krull-Schmidt theorem. 


$$
\begin{aligned}
{[\alpha(H):(e)] } & \leqq[A(H):(e)][B(H):(e)] \\
& =[H: \operatorname{ker} A][H: \operatorname{ker} B] \\
& \leqq \operatorname{Max}\left\{[H: \operatorname{ker} A]^{2},[H: \operatorname{ker} B]^{2}\right\} \\
& <[H:(e)]
\end{aligned}
$$

which is absurd. This shows that $B$ is an isomorphism of $H$ onto $K$. Similarly, we can show by the help of the fact $H=A(H) C(K)$, a consequence of (1.1.7), that $C$ is an isomorphism of $K$ onto $H$. From the assumption that $\alpha=\left[\begin{array}{ll}A & C \\ B & D\end{array}\right] \in \mathfrak{X}(G)$ with $D \notin \mathfrak{X}(K)$, we derive the same conclusions. Consequently, we see that if $\left[\begin{array}{ll}A & C \\ B & D\end{array}\right] \in \mathfrak{A}(G)$ then either

(i ) $A \in \mathfrak{A}(H)$ and $D \in \mathfrak{X}(K)$ or

(ii) $B$ is an isomorphism of $H$ onto $K$, and $C$ is an isomorphism of $K$ onto $H$.

Suppose $\left[\begin{array}{ll}A & C \\ B & D\end{array}\right] \in \mathfrak{U}(G)$ with (i). Then $\left[\begin{array}{cc}A & 0 \\ 0 & D\end{array}\right] \in \mathfrak{U}(G)$, and therefore

$$
\left[\begin{array}{ll}
A & C \\
B & D
\end{array}\right]\left[\begin{array}{cc}
A & 0 \\
0 & D
\end{array}\right]^{-1}=\left[\begin{array}{cc}
E & C D^{-1} \\
B A^{-1} & E
\end{array}\right] \in \mathfrak{A}(G) \text {. }
$$

Applying (1.1.5) to $\left[\begin{array}{cc}E & C D^{-1} \\ B A^{-1} & E\end{array}\right]$, we can show that $B=0$. On the other hand, we have $C=0$ by (1.1.6). Similarly, we have $A=0$ and $D=0$ in case (ii). Consequently, $\mathfrak{A}(G)$ is the totality of all quadruples of the following two types:

( I) $\left[\begin{array}{cc}A & 0 \\ 0 & D\end{array}\right]$ where $A \in \mathfrak{A}(H)$ and $D \in \mathfrak{A}(K)$.

(II) $\left[\begin{array}{ll}0 & C \\ B & 0\end{array}\right]$ where $B$ is an isomorphism of $H$ onto $K$ and $C$ is an isomorphism of $K$ onto $H$.

Let $\widetilde{I}_{2}$ be the subgroup of $\mathfrak{U}(G)$ generated by $\left[\begin{array}{cc}0 & C_{1} \\ B_{1} & 0\end{array}\right]$ where $B_{1}$ and $C_{1}$ are defined by $B_{1}(h, e)=\left(h^{-1},\langle h\rangle_{H}\right)$ and $C_{1}=B_{1}^{-1}$. $\mathfrak{A}(G)$ is a splitting extension of $\mathfrak{A}_{H}(G)$ by $\widetilde{I}_{2}$. Using (3.3), we conclude the proof.

As an application we mention

THEOREM 3.6. Let $G$ be the holomorph of the symmetric group $S_{n}$ of degree $n$ where $n \geqq 3, n \neq 4$ and $n \neq 6$. Then, $G$ is centerless, every automorphism of $G$ which maps $S_{n} \subset G$ onto itself is inner, and $\mathfrak{U}(G)$ is a splitting extension of $\mathfrak{\Im}(G)$ by a group of order 2 .

4. Application to the holomorph $(H, K ; \iota)$ of an abelian group $H$ with respect to an abelian subgroup $K$ of $\mathfrak{A}(H)$. In this section, we use the additive notation for the composition in the abelian group $H$ and the usual notation for each of the compositions in the ring of endomorphisms of $H$. Thus, e.g., by 2 is meant the mapping which carries $h$ 
into $2 h$. In case $H$ is abelian, $\mathfrak{C}^{r}=\sqrt{ }, \mathfrak{P}^{s}=\mathfrak{P}^{r}=\mathfrak{P}$. In the first seven propositions, we do not assume that $K$ is abelian. Very quickly we can verify

Proposition 4.1. Let $G=(H, K ; \iota)$ where $H$ is abelian. Suppose that there exists $k_{0} \in Z(K)$ such that $k_{0}$ has no non-trivial fixed point. Let $U \in \mathbb{E}$. Then $U \in \mathfrak{P}$ if and only if $U\left(k_{0}\right) \in\left(1-k_{0}\right) H$.

By (4.1) and (1.2), we have

Proposition 4.2. Let $G=(H, K ; \iota)$ where $H$ is abelian. Suppose that there exists $k_{0} \in Z(K)$ such that $1-k_{0}$ is an automorphism of $H$. Then $\mathfrak{E}=\mathfrak{P}$.

By (4.2), (2.11) and (2.13), we have a well-known result:

Proposition 4.3." Let $G$ be the holomorph of an abelian group $H$, and let the mapping 2 be an automorphism of $H$. Then $\mathfrak{A}_{H}(G)=\mathfrak{\Im}(G)$.

By (1.1.6), we have

Proposition 4.4. Let $G=(H, K: \iota)$ where $H$ is abelian. Then $\left[\begin{array}{cc}A & C \\ 0 & D\end{array}\right] \in \mathfrak{A}_{H}(G)$ implies that $A \in N(K, \mathfrak{U}(H))$.

It is easy to verify

Proposition 4.5. Let $G=(H, K ; \iota)$ where $H$ is abelian. Suppose that there exists $k_{0} \in Z(K)$ such that $k_{0}$ has no non-trivial fixed point. Let $h_{0}$ be any element of $H$. Then there exists at most one $U \in \mathbb{5}$ such that $U\left(k_{0}\right)=h_{0}$.

By $(1-K) H$, we denote the set of all elements of the form $(1-k) h$ for some $k \in K$ and some $h \in H$.

Proposition 4.6. Let $G=(H, K ; \iota)$ where $H$ is abelian and $(1-K) H$ is a subgroup of $H$. Suppose that there exists $k_{0} \in Z(K)$ such that $1-k_{0}$ is an isomorphism of $H$ onto $(1-K) H$. Let $h_{0}$ be any fixed element of $H$. Then there exists exactly one $U \in \mathbb{E}$ such that $U\left(k_{0}\right)=h_{0}$.

Proof. Define $U(k)=\left(1-k_{0}\right)^{-1}(1-k)\left(h_{0}\right)$ to show the existence. The uniqueness follows from (4.5).

Theorem 4.7. Let $G=(H, K ; \iota)$ where $H$ is abelian and $(1-K) H$ is a subgroup of $H$. Suppose that there exists $k_{0} \in Z(K)$ such that $1-k_{0}$ is an isomorphism of $H$ onto $(1-K) H$. Then $\mathfrak{A}_{H}(G)$ is isomorphic to a splitting extension of $H$ by $N(K, \mathfrak{A}(H))$. If further we assume $K \subset Z(N(K, \mathfrak{Y}(H)))$, then

4 See [5, p. 617]. 


$$
\mathfrak{N}_{H}(G) \cong(H, N(K, \mathfrak{A}(H)) ; \iota) .
$$

Proof. By (4.4) and (2.7), we see that

$$
\mathfrak{A}_{H}(G) \cong(\mathfrak{S}, N ; \theta)
$$

with $\theta \in \operatorname{Hom}(N, \mathfrak{A}(\mathfrak{E}))$ defined by $\theta_{A}(U)=A U\left\langle A^{-1}\right\rangle_{N} / K$ for $A \in N$ and $U \in \mathbb{E}$. By (4.6), the mapping $U \rightarrow U\left(k_{0}\right)$ is an isomorphism of $\mathbb{C}$ onto $H$. Hence the former statement. To prove the latter part, we observe that $\left\langle A^{-1}\right\rangle_{N} / K=E$ and therefore $\theta_{A}(U)=A U$. The mapping $(U, A) \rightarrow$ $\left(U\left(k_{0}\right), A\right)$ of $(\sqrt{5}, N ; \theta)$ into $(H, N ; \iota)$ establishes $(4.7 .1)$.

As an immediate consequence we have

Corollary 4.8. Let $G=(H, K ; \iota)$ where $H$ and $K$ are abelian and $(1-K) H$ is a subgroup of $H$. Suppose that there exists $k_{0} \in K$ such that $1-k_{0}$ is an isomorphism of $H$ onto $(1-K) H$. Suppose also that $N(K, \mathfrak{U}(H))=K$. Then

$$
\mathfrak{U}_{H}(G) \cong G \text {. }
$$

Theorem 4.9. Let $G=(H, K ; \iota)$ where $H$ and $K$ are abelian and $(1-K) H$ is a subgroup of $H$. Suppose that there exists $k_{0} \in K$ such that $1-k_{0}$ is an isomorphism of $H$ onto $(1-K) H$. Then

$$
\Im(G) \cong G .
$$

Proof. Let $\left[\begin{array}{cc}A & C \\ 0 & D\end{array}\right] \in \Im(G)$. Then $A \in K$, and therefore $D=E$ by (1.1.6). A $A \in K$ also implies that $\left[\begin{array}{cc}A & 0 \\ 0 & E\end{array}\right] \in \mathfrak{\Im}(G)$. Hence

$$
\left[\begin{array}{cc}
E & C \\
0 & E
\end{array}\right]=\left[\begin{array}{cc}
A & C \\
0 & E
\end{array}\right]\left[\begin{array}{cc}
A & 0 \\
0 & E
\end{array}\right]^{-1} \in \Im(G) \text {. }
$$

Therefore by (2.8) $C \in \mathfrak{P}$. Conversely, suppose we are given $A \in K$ and $C \in \mathfrak{P}$. By (2.9), there exists a unique $\left[\begin{array}{cc}E & C_{1} \\ 0 & D_{1}\end{array}\right] \in \Im(G)$ such that $C_{1} D_{1}^{-1}=C$. By (2.3.3) $D_{1}=E$ and therefore $C_{1}=C$. Hence

$$
\left[\begin{array}{cc}
A & C \\
0 & E
\end{array}\right]=\left[\begin{array}{cc}
E & C \\
0 & E
\end{array}\right]\left[\begin{array}{cc}
A & 0 \\
0 & E
\end{array}\right] \in \Im(G) \text {. }
$$

Thus we have shown that $\Im(G)$ is the totality of all $\left[\begin{array}{cc}A & C \\ 0 & E\end{array}\right]$ with $A \in K$ and $C \in \mathfrak{P}$. By (4.1), $\left(1-k_{0}\right)^{-1} C\left(k_{0}\right)$ can be defined for each $C \in \mathfrak{P}$. Define a mapping

$$
\left[\begin{array}{cc}
A & C \\
0 & E
\end{array}\right]: \rightarrow\left(\left(1-k_{0}\right)^{-1} C\left(k_{0}\right), A\right)
$$


of $\Im(G)$ into $G$. By (4.1), (4.5) and (4.6), we see that the mapping is an isomorphism of $\mathfrak{\Im}(G)$ onto $G$.

THEOREM 4.10. Let $G=(H, K ; \iota)$ where $H$ and $K$ are abelian and $(1-K) H$ is a subgroup of $H$. Suppose that there exists $k_{0} \in K$ such that $1-k_{0}$ is an isomorphism of $H$ onto $(1-K) H$. Suppose also that $N(K, \mathfrak{A}(H))=K$. Then

$$
\frac{\mathfrak{A}_{H}(G)}{\mathfrak{I}(G)} \cong \frac{G}{\left(\left(1-k_{0}\right) H, K ; \iota^{\prime}\right)}
$$

where $\iota_{k}^{\prime}, k \in K$, is the automorphism of $\left(1-k_{0}\right) H$ obtained by restricting $k$ to $\left(1-k_{0}\right) H$.

Proof. By (1.1.6), (2.1), (2.3) and (2.6), we see $\mathfrak{A}_{H}(G)$ is the totality of all $\left[\begin{array}{cc}A & C \\ 0 & E\end{array}\right]$ with $A \in K$ and $C \in \mathbb{E}$, while $\Im(G)$ is the totality of all $\left[\begin{array}{cc}A & C \\ 0 & E\end{array}\right]$ with $A \in K$ and $C \in \mathfrak{P}$ as we saw in the proof of (4.9). Under the mapping

$$
\left[\begin{array}{cc}
A & C \\
0 & E
\end{array}\right] \rightarrow\left(C\left(k_{0}\right), A\right)
$$

which establishes $\mathfrak{U}_{H}(G) \cong(H, K ; \iota)$, a particular case of (4.7.1), the image of $\mathfrak{\Im}(G)$ is $\left(\left(1-k_{0}\right) H, K ; \iota^{\prime}\right)$ by $(4.1)$.

Theorem 4.11. Let $G=(H, K ; \iota)$ where $H$ and $K$ are abelian and $(1-K) H$ is a subgroup of $H$. Suppose that $-1 \in K$ and that 2 is an isomorphism of $H$ onto $(1-K) H$. Suppose also that $N(K, \mathfrak{A}(H))=K$. If $[H: 2 H]=2$, then $\mathfrak{A}_{H}(G)$ is a splitting extension of $\mathfrak{\Im}(G)$ by a group of order 2.

Proof. Take $h_{0} \in H, h_{0} \notin 2 H$. By (4.6) there exists $C_{0} \in \mathbb{E}$ such that $C_{0}(-1)=h_{0}$. By the proof of the previous theorem, $\left[\begin{array}{cc}-E & C_{0} \\ 0 & E\end{array}\right] \notin \Im(G)$. It is easy to see that $\mathfrak{A}_{H}(G)$ is a splitting extension of $\mathfrak{\Im}(G)$ by the subgroup $\widetilde{I}_{2}$ generated by $\left[\begin{array}{cc}-E & C_{0} \\ 0 & E\end{array}\right]$.

As an application of the discussion in this section we prove

THEOREM 4.12. Let $G$ be the holomorph of a non-zero subgroup $H$ of the additive group $R$ of rational numbers. Then

$$
\mathfrak{A}(G)=\mathfrak{A}_{H}(G) \cong \mathfrak{J}(G) \cong G .
$$

In case 2 is an automorphism of $H, \mathfrak{A}(G)=\Im(G)$. In case 2 is not an 
automorphism of $H, \mathfrak{A}(G)$ is a splitting extension of $\mathfrak{\Im}(G)$ by a group of order 2.

Proof. The mapping 2 is an isomorphism of $H$ onto

$$
2 H=(1-\mathfrak{U}(H)) H .
$$

Hence, by (4.8) and (4.9), we have

$$
\mathfrak{A}_{H}(G) \cong G \cong \Im(G) .
$$

If $2 \in \mathfrak{A}(H)$, then $\mathfrak{A}_{H}(G)=\mathfrak{\Im}(G)$ by (4.3). On the other hand, if $2 \notin \mathfrak{A}(H)$, then $[H: 2 H]=2$ and therefore $\mathfrak{A}(G)$ is a splitting extension of $\mathfrak{\Im}(G)$ by a group of order 2 by (4.11). It remains to show that $\mathfrak{A}(G)=\mathfrak{A}_{H}(G)$. Let $\alpha=\left[\begin{array}{ll}A & C \\ B & D\end{array}\right] \in \mathfrak{A}(G)$. Then by (1.1.2) $B \in \operatorname{Hom}(H, \mathfrak{U}(H))$. Therefore $H /$ ker $B \cong B(H) \subset \mathfrak{U}(H)$. Since $H$ cannot be embedded isomorphically into $\mathfrak{A}(H)$, we have $\operatorname{ker} B \neq(0)$. Hence $B(H)$ is a periodic subgroup of $\mathfrak{U}(H)$, and therefore $B(H)=(1)$ or $B(H)=(1,-1)$. In case $2 \in \mathfrak{U}(H)$, $H$ has no subgroup of index 2, and therefore $B(H)=(1)$. Suppose that $2 \notin \mathfrak{A}(H)$. In order to prove that $B(H)=(1)$, let us suppose on the contrary that $B(H)=(1,-1)$. Let $s$ be the smallest positive integer in $H$. Suppose that 2 is not a prime factor of $s$. Let $\lambda$ be the nonnegative integer such that $s / 2^{\lambda} \in H$ and that $s / 2^{\lambda+1} \notin H$. Every element of $H$ can be written in the form $s n_{1} / 2^{\lambda} n_{2}$ with $\left(n_{2}, 2\right)=1$, and $s n_{1} / 2^{\lambda} n_{2} \in H$ is an element of $\operatorname{ker} B$ if and only if $n_{1}$ is even. Write $n_{1}=2^{\mu} n_{3}$ with $\left(n_{3}, 2\right)=1$. By induction on $\mu$ we can show, by means of (1.1.1), that $A(h)=0$ for every $h \in \operatorname{ker} B$. By (1.1.1) we also have $A(h)=A\left(s / 2^{\lambda}\right)$ for every $h \notin \operatorname{ker} B$. Consequently $\alpha(h)=(A(h), B(h))$ assumes at most four different values as $h$ ranges over $H$, which is absurd. In case 2 is a prime factor of $s$, let $-\lambda$ be the number of the factor 2 in the prime factorization of $s$. The same argument goes through and we have $B(H)=(1)$ in any case. This proves that $\mathfrak{A}(G)=\mathfrak{U}_{H}(G)$.

As an immediate consequence of (4.12) we have

COROLlaRY $4.13 .^{5}$ Let $G$ be the holomorph of a non-zero subgroup of the additive group $R$ of rational numbers. Then $G$ is complete if and only if 2 is an automorphism of $H$.

\section{REFERENCES}

1. Yu. A. Gol'fand, On the group of automorphisms of the holomorph of a group, Rec. Math. (Math. Sbornik) N. S., 27 (69) (1950), 333-350.

2. A. G. Kurosh, Theory of groups I, II, 2nd ed., New York, 1955-1956.

3. G. A. Miller, On the multiple holomorph of a group, Math. Ann., 66 (1908), 133-142.

5 Cf. [5, p. 619]. 
4. W. H. Mills, The automorphisms of the holomorph of a finite abelian group, Trans. Amer. Math. Soc., 85 (1957), 1-34.

5. W. Peremans, Completeness of holomorphs, Koninkl. Nederl. Akad. Proc. Ser. A, 60 (1957), 608-619.

6. W. Spect, Gruppentheorie, Berlin, 1956.

INTERNATIONAL BUSINESS MACHINES CORPORATION, YORKTOWN HEIGHTS, N.Y. 


\title{
PACIFIC JOURNAL OF MATHEMATICS
}

\section{EDITORS}

\author{
Ralph S. Phillips \\ Stanford University \\ Stanford, California \\ F. H. BrownelL \\ University of Washington \\ Seattle 5 , Washington
}

A. L. Whiteman

University of Southern California

Los Angeles 7. California

L. J. PAIGE

University of California

Los Angeles 24, California

\section{ASSOCIATE EDITORS}

\author{
E. F. BECKENBACH \\ T. M. CHERRY
} \\ D. DERRY \\ M. OHTSUKA \\ UNIVERSITY OF BRITISH COLUMBIA \\ CALIFORNIA INSTITUTE OF TECHNOLOGY \\ UNIVERSITY OF CALIFORNIA \\ MONTANA STATE UNIVERSITY \\ UNIVERSITY OF NEVADA \\ NEW MEXICO STATE UNIVERSITY \\ OREGON STATE COLLEGE \\ UNIVERSITY OF OREGON \\ OSAKA UNIVERSITY \\ UNIVERSITY OF SOUTHERN CALIFORNIA
}

H. L. ROYDEN

E. SPANIER
E. G. STRAUS

F. WOLF

\section{SUPPORTING INSTITUTIONS}

\author{
STANFORD UNIVERSITY \\ UNIVERSITY OF TOKYO \\ UNIVERSITY OF UTAH \\ WASHINGTON STATE COLLEGE \\ UNIVERSITY OF WASHINGTON \\ AMERICAN MATHEMATICAL SOCIETY \\ CALIFORNIA RESEARCH CORPORATION \\ HUGHES AIRCRAFT COMPANY \\ SPACE TECHNOLOGY LABORATORIES \\ NAVAL ORDNANCE TEST STATION
}

Mathematical papers intended for publication in the Pacific Journal of Mathematics should be typewritten (double spaced), and the author should keep a complete copy. Manuscripts may be sent to any one of the four editors. All other communications to the editors should be addressed to the managing editor, L. J. Paige at the University of California, Los Angeles 24, California.

50 reprints per author of each article are furnished free of charge; additional copies may be obtained at cost in multiples of 50 .

The Pacific Journal of Mathematics is published quarterly, in March, June, September, and December. The price per volume (4 numbers) is $\$ 12.00$; single issues, $\$ 3.50$. Back numbers are available. Special price to individual faculty members of supporting institutions and to individual members of the American Mathematical Society: $\$ 4.00$ per volume; single issues, $\$ 1.25$.

Subscriptions, orders for back numbers, and changes of address should be sent to Pacific Journal of Mathematics, 103 Highland Boulevard, Berkeley 8, California.

Printed at Kokusai Bunken Insatsusha (International Academic Printing Co., Ltd.), No. 6 , 2-chome, Fujimi-cho, Chiyoda-ku, Tokyo, Japan.

PUBLISHED BY PACIFIC JOURNAL OF MATHEMATICS, A NON-PROFIT CORPORATION

The Supporting Institutions listed above contribute to the cost of publication of this Journal, but they are not owners or publishers and have no responsibility for its content or policies. 


\section{Pacific Journal of Mathematics}

\section{Vol. 11, No. $3 \quad$ BadMonth, 1961}

Errett Albert Bishop, A generalization of the Stone-Weierstrass theorem ..........

Hugh D. Brunk, Best fit to a random variable by a random variable measurable with

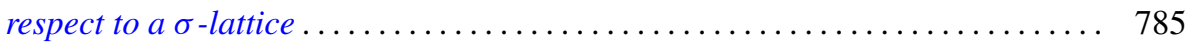

D. S. Carter, Existence of a class of steady plane gravity flows . . . . . . . . . . 803

Frank Sydney Cater, On the theory of spatial invariants ............... 821

S. Chowla, Marguerite Elizabeth Dunton and Donald John Lewis, Linear

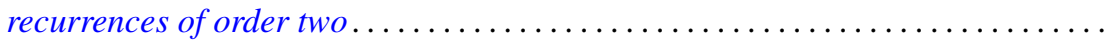

Paul Civin and Bertram Yood, The second conjugate space of a Banach algebra as

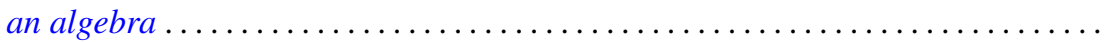

William J. Coles, Wirtinger-type integral inequalities .

Shaul Foguel, Strongly continuous Markov processes ....................

David James Foulis, Conditions for the modularity of an orthomodular lattice ...... Jerzy Górski, The Sochocki-Plemelj formula for the functions of two complex variables.

John Walker Gray, Extensions of sheaves of associative algebras by non-trivial

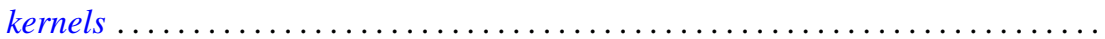

Maurice Hanan, Oscillation criteria for third-order linear differential equations .... 919 Haim Hanani and Marian Reichaw-Reichbach, Some characterizations of a class of unavoidable compact sets in the game of Banach and Mazur .............

John Grover Harvey, III, Complete holomorphs . . . . . . . . . . . . . . . . 961 Joseph Hersch, Physical interpretation and strengthing of M. Protter's method for vibrating nonhomogeneous membranes; its analogue for Schrödinger's

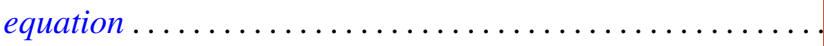

James Grady Horne, Jr., Real commutative semigroups on the plane...

Nai-Chao Hsu, The group of automorphisms of the holomorph of a group...

F. Burton Jones, The cyclic connectivity of plane continua

John Arnold Kalman, Continuity and convexity of projections and barycentric

coordinates in convex polyhedra

Samuel Karlin, Frank Proschan and Richard Eugene Barlow, Moment inequalities of

Pólya frequency functions .

Tilla Weinstein, Imbedding compact Riemann surfaces in 3-space. .

Azriel Lévy and Robert Lawson Vaught, Principles of partial reflection in the set

theories of Zermelo and Ackermann

Donald John Lewis, Two classes of Diophantine equations .....

Daniel C. Lewis, Reversible transformations . . .

Gerald Otis Losey and Hans Schneider, Group membership in rings and

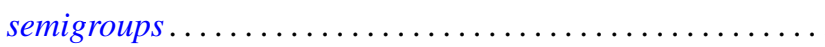

M. N. Mikhail and M. Nassif, On the difference and sum of basic sets of

polynomials.

Alex I. Rosenberg and Daniel Zelinsky, Automorphisms of separable algebras .

Robert Steinberg, Automorphisms of classical Lie algebras .... 